\title{
Problème de la commercialisation du bétail en Afrique
}

\author{
por M. LACROUTS
}

\begin{abstract}
SOMMAIRE
Dans cette communication sonl trattés les divers aspects concernant le commerce du gros bétail dans l'Afrique de l'Ouest, dont la zone de production est la zone Sohélienne ef les centres consommateurs les pays le long de l'océan Atlantique (du Sénégal à la Républıque Centrafricaine). La relation entre les régions productrices el les grandes villes dans les régıons du Cameroun et de Madagascar esł aussi mentionnée.

Le plus grand nombre des animaux en Afr que Centrale tropicale est élevé dans les zones les plus septentrionales, c'est-à-dıre dans des régions qui seraient totalement désertées par l'homme sans cette actıvité pastorale.

Dans le tablecu No 1 les effectifs du bétail sont donnés pour les différents pays de production en relation avec la densité de la population. On constale la plus faible densité bovıne dans les pays de niveau de vie le plus élevé (Côte-d'lvorre ef Ghana).

Quant aux modalités du commerce, la situation existante est que le problème des transports des anımaux vers les centres de consommation domıne souvent l'économie des systèmes.

Les tableaux Nos 4 et 5 donnent une indication des frass de commercialisation par les deux moyens principaux de transport : acheminement à pieds et transport par camion, entre lesquels presqu'aucune différence n'existe pour le prix du bétall rendu dans la zone de consommation.

L'intérêt du transport moderne consiste dans l'économie de substance noble, ce qui est très important, mais cela ne permet pas de payer mieux le producteur ou d'abaisser le prix de la viande chez le consommateur Pour l'avenır, le développement de la production de viande co lavoir une priorité absolve. Pour cela de grands problèmes doivent être résolus, dont les plus importants sont : la technıque d'élevage, l'amélıoration de qualité du bétaıl ef des mayens de transport, l'amélıoratıon des modalıtés commerc ales combınée avec une organısation ralıonnelle des circuits de vente de la $v$ ande, accompagnée des facilités nécessaires de paiement du bétall sur les marchés des centres de consommatıan.
\end{abstract}

Le commerce du bétall en Afrique a pour ojet, comme partout alleurs, la prospecion des zones de production, l'acheminement des anımaux et

* Communication au 2 e sémınare international sur l'étude des zones de pâturages naturels 17-22 avril 1967. Repris de la publication 524 de l' " ITC-UNESCO centre for integrated surveys ». Delft Netherlands. leur vente dans les centres consommateurs, maıs le caractère partıculıer et l'orıgınalité de ces opérations, sur le contınent africaın, sont liés au contexte géographique et humaın du milieu dans lequel elles se déroulent.

Nous décrirons donc rapidement les conditions de la production afin d'appréhender ef de 
comprendre mieux les modalités exactes de ce commerce. En effet, c̀ l'occasion des nombreuses missions que nous avons effectuées, nous avons pu nous rendre compte combien d'idées absolument aberrantes étaient propagées, solt par des technıciens ou économistes, connaissant mal ces régions, solt même par les autorités responsables de ces pays. Ces confusions sont parfols lourdes de conséquences, car elles conduisent à proposer des solutions sans efficacité, souvent dangereuses ef toujours mal adaptées aux problèmes à résoudre.

Nous tratterons $\mathrm{lal}$ du commerce du gros bétall dans l'Afrique de l'Ouest, entre d'une part la zone sahélienne groupant le Nord Sénégal, la Mauritanie, le Mali, la Haute Volta, le Niger et le Tchad, et, d'autre part, les pays consommateurs comprenant le Sud Sénégal, le Libéria, la Côte-d'Ivoire, le Ghana, le Nigeria, la République Centrafricaıne. Cette analyse s'applıque également à la commercialisation entre le Nord et le Sud du Cameroun, ainsi qu'à Madagascar, entre les régions productrices et les grandes villes.

\section{I. - LES CARACTÉRISTIQUES PRINCIPALES DE LA PRODUCTION}

Dans les régions de grand élevage, celui-ci est partout de type exiensif, les animaux sont des zébus appartenant à des pasteurs transhumants : Maures, Peuhls, et Bororos ou arabes. A Madagascar, quaique les éleveurs soient presque tous sédentarisés, les troupeaux sont également soumıs à des déplacements d'amplıtudes plus faibles. Les transhumances sont une remarquable adaptation à un milieu diffichle, elles permettent de réaliser un équilıbre, souvent instable, entre la satısfaction de besoins en eau et en pâturages. Leur maintien est indispensable d la survie et au développement du troupeau bovin dans le Sahel.

Cet élevage est particulièrement important sur le plan humaın, puisqu'il est souvent l'unique ressource des populations occupant ces zones les plus septentrionales de l'Afrique tropicale. Sans cette actıvité et ce mode de vie imposés par la conduite du cheptel, toute cette région serait désertée par l'homme. Economiquement, l'actvité pastorale jove un rôle essentiel puisque sa production est vitale pour approvisionner en viande les nombreuses populations des pays côtiers.

Un autre aspect de cet élevage, influençant directement l'économie du système, c'est la petite taille de l'unité de production, contralrement à ce que laisse supposer le terme classique d'élevage extensıf, qui lui est applıqué, on rencontre fort peu de «grands propriétares». Le Peuhl, le Maure, le chef Touareg, aux milliers d'animaux, dorvent aujourd'hui être classés dans les personnages de légende, même s'ils existent, Ils ne sont plus représentatifs. La grande majorité des pasteurs ne possède guère plus de 40 à 80 têtes de bovins. Compte tenu de l'évolution des besorns, du renchérissement des prodults essentiels, ces éleveurs sont de plus en plus obligés de vendre jeunes leurs anumaux mâles. Appliquée à des races fort peu précoces, cette politique aboutıt à la commercialisation ef souvent d̀ l'abattage d'animaux n'ayant pas atteınt leur plein développement.

\section{Evaluation de la production.}

La production d'un troupeau est fonction de l'effectif (le capital) et de sa productıvité (taux d'explostation), enfin son développement dépendra de l'évolution respective de ces deux termes.

Nous présentons dans le tableau No 1 une évaluation de l'ımportance du cheptel bovin en 1966, dans les différents pays envisagés. Nous distinguons les deux grands types d'anımaux : les zébus de la zone sahélıenne, et les taurıns trypanotolérants des régions soudano-guineennes. De même, on peut classer les pays en deux groupes avec d'une part les pays excédentaires en viande bovine (appartenant à la zone sahélienne) ef d'autre part les pays déficitarres.

Au total, l'Afrique de l'Ouest possède un cheptel bovin de 33 millions de têtes dont la répartition est très inégale. La densité d'anımaux par rapport à la population varie de façon énorme entre les extrêmes de 6 et de 250 têtes de bovins par cent habitants. Ces Inégalités explıqueront un volume d'échange de produits de l'élevage très important et proportionnellement peut-être supérieur à celui observé dans. les zones économiques plus développées.

Les pays excédentaires, exportateurs net, groupent 17.300 .000 têtes pour 15.850 .000 habltants, soit une densité moyenne de l'ordre de 1,10 tête par habitant. L'autre groupe de pays 
TABLEAU No 1

Evaluation du Cheptel bovin (1) dans différents pays de 'Afrique de l'Ouest et à Madagascar

\begin{tabular}{|c|c|c|c|c|c|}
\hline Pays & Cheplel Zébus & Cheple, Taurins & Total & Nbre d'habitants & $\begin{array}{l}\text { Nbre de bovins } \\
\text { p. } 100 \text { habilants }\end{array}$ \\
\hline 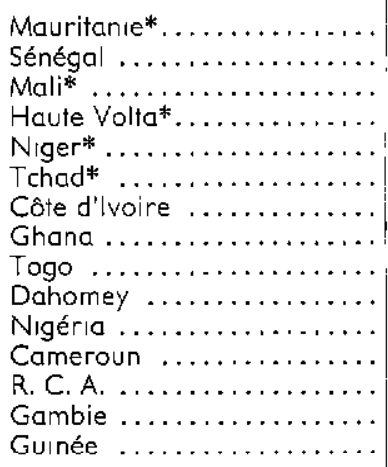 & $\begin{array}{c}2.000 .000 \\
1500000 \\
3.800 .000 \\
1.500 .000 \\
4000000 \\
5000000 \\
- \\
= \\
- \\
7.000000 \\
1.800000 \\
440.000 \\
-\end{array}$ & $\begin{array}{r}700 \\
500 \\
500 \\
- \\
- \\
350000 \\
500000 \\
180.000 \\
400000 \\
1000000 \\
300.000 \\
10000 \\
220000 \\
1500000\end{array}$ & $\begin{array}{r}2.000000 \\
2200000 \\
4.300 .000 \\
2.000000 \\
4000000 \\
5000000 \\
350.000 \\
500.000 \\
180000 \\
400.000 \\
8.000 .000 \\
2100000 \\
450000 \\
220.000 \\
1.500 .000\end{array}$ & $\begin{array}{r}800.000 \\
3.300 .000 \\
4.300 .000 \\
4.550000 \\
3300.000 \\
2.900 .000 \\
4.000 .000 \\
7.800 .000 \\
1.500 .000 \\
2.300 .000 \\
45000.000 \\
3700.000 \\
1.400 .000 \\
350.000 \\
3300.000\end{array}$ & $\begin{array}{r}250 \\
67 \\
100 \\
44 \\
121 \\
172 \\
9 \\
6 \\
12 \\
17 \\
18 \\
57 \\
32 \\
63 \\
36\end{array}$ \\
\hline Total Afrique Ovest ....... & 27.040 .000 & 6.160 .000 & 33200.000 & 88.500 .000 & 37 \\
\hline Madagascar...$\ldots \ldots \ldots$ & 10500.000 & - & 10.500 .000 & 5.800000 & 181 \\
\hline
\end{tabular}

* Pays excédentaires, exportateurs net de bétali et de viande.

(1) Données valables pour 1965-1966.

possède un cheptel respectable de 15.900 .000 têtes, mais dont la densité par rapport à une population de 71.200 .000 habitants est 5 fois moins importante (22 têtes pour 100 habitants). Enfin, ce sont les pays où le niveau de vie est le plus élevé qui ont la plus faible densité bovine (Côte-d'ivorre et Ghana).

\section{Détermination des taux de production et d'exploi- tation.}

Ce problème est très complexe, car le manque de données suffisantes sur la production et l'évolution du cheptel, oblıge à l'aborder par le bials de la structure du troupeau. C'est ce que nous avons tenté dans différentes études. Grâce d̀ des compositions de troupeaux observées et à l'utilisation des divers facteurs essentıels (fécondité, précocité, mortalıté naturelle aux différents âges), fournis par les recherches zootechniques et vérifiés par interrogatores, nous essayons de construire des structures par âge, valables pour des zones homogènes, et ensulte d'en déduire des taux plausibles de production, d'exploitation et d'évolution.
Bien que cette méthode soit précise dans sa conception, les résultats obtenus ne doivent être considérés que comme des ordres de grandeur. car les rares données statıstıques disponibles sont souvent sujettes à caution et, par alleurs, les facteurs essentiels qu régıssent la dynamique du troupeau sont parfois difficiles à cerner avec précision et peuvent en outre varier assez consıdérablement dans le temps.

Dans le tableau $n^{\circ} 2$, nous présentons quelques exemples de taux de production et d'exploitation extraits d'études récentes.

Les données obtenues à partır des effectıfs et des taux de production sont complétées par les enquêtes menées sur la commercialisation et la consommation. On arrive ainsı à donner par zone, pays ou région, des tableaux ressources emplois, dont nous présentons $I C \mid$ un exemple relatıf à l'ensemble du troupeau malien (tableau No 3). Ces productions ef utilisations sont obtenues en nombre d'anımaux, on peut facilement, grâce à l'utilısation de poids moyen, par catégorie, obtenir des données en tonnes de viande carcasse. On peut égatement convertır l'ensem- 
TABLEAU No 2

Exemples de taux de production et d'exploitation dans différents pays africoins ef malgaches

Unités : pourcentage par rapport à l'effectif tolal.

\begin{tabular}{|c|c|c|c|c|c|}
\hline \multirow{2}{*}{ Types d'animaux } & \multicolumn{2}{|c|}{ Cameraun } & \multicolumn{2}{|c|}{ Malı } & \multirow{2}{*}{$\begin{array}{c}\text { Madagascar } \\
\text { (moyenne } \\
\text { nationale) }\end{array}$} \\
\hline & $\begin{array}{l}\text { Régıon } \\
\text { de Adamaoua }\end{array}$ & $\begin{array}{l}\text { Région } \\
\text { Nord }\end{array}$ & $\begin{array}{l}\text { Troupeau } \\
\text { Zébus }\end{array}$ & $\begin{array}{c}\text { Troupeau } \\
\text { Taurins }\end{array}$ & \\
\hline $\begin{array}{l}\text { Vaches de réforme...... } \\
\text { Vaches stériles.......... } \\
\text { Mâles adultes } \ldots \ldots \ldots \ldots \\
\text { Taurıllons } \ldots \ldots \ldots \ldots\end{array}$ & $\begin{array}{l}3 \\
2,2 \\
5,1 \\
1,2\end{array}$ & $\begin{array}{l}4 \\
1 \\
6,4\end{array}$ & $\begin{array}{l}4,2 \\
0,8 \\
4,1 \\
2,9\end{array}$ & $\begin{array}{l}4,5 \\
0,9 \\
2,9 \\
4,7\end{array}$ & $\begin{array}{l}3,1 \\
1 \\
5\end{array}$ \\
\hline Taux d'exploitation total. & 11,5 & 11,4 & 12 & 13 & 9,1 \\
\hline Génisses capitalisées .... & - & 1,8 & 3 & 3,7 & 1 \\
\hline Taux de production tolal. & 11,5 & 13,2 & 15 & 16,7 & 10,1 \\
\hline
\end{tabular}

TABLEAU No 3

Bilan — Ressources — Emplois de la production au Mali en 1964-1965

Unıtés : tête de bétail tonne de carcasse.

\begin{tabular}{|c|c|c|c|c|c|c|c|c|c|c|}
\hline \multirow{3}{*}{$\begin{array}{l}\text { Catégorie } \\
\text { d'anımaux }\end{array}$} & \multicolumn{4}{|c|}{ Ressources } & \multirow{2}{*}{\multicolumn{2}{|c|}{$\begin{array}{c}\text { Total Ressources } \\
\text { el Emplois }\end{array}$}} & \multicolumn{4}{|c|}{ Emplois } \\
\hline & \multicolumn{2}{|c|}{$\begin{array}{c}\text { Production (1) } \\
\text { nationale }\end{array}$} & \multicolumn{2}{|c|}{ Importations } & & & \multicolumn{2}{|c|}{$\begin{array}{l}\text { Consommation } \\
\text { intérieure }\end{array}$} & \multicolumn{2}{|c|}{ Exportation } \\
\hline & $\begin{array}{l}\text { Nombre } \\
\text { de têtes }\end{array}$ & $\begin{array}{l}\text { Ton- } \\
\text { nage }\end{array}$ & $\begin{array}{l}\text { Nombre } \\
\text { de têtes }\end{array}$ & $\begin{array}{l}\text { Ton } \\
\text { nage }\end{array}$ & $\begin{array}{l}\text { Nombre } \\
\text { de têtes }\end{array}$ & $\begin{array}{l}\text { Ton- } \\
\text { nage }\end{array}$ & $\begin{array}{l}\text { Nombre } \\
\text { de têtes }\end{array}$ & $\begin{array}{l}\text { Ton- } \\
\text { nage }\end{array}$ & $\begin{array}{l}\text { Nombre } \\
\text { de têtes }\end{array}$ & $\begin{array}{l}\text { Ton- } \\
\text { nage }\end{array}$ \\
\hline $\begin{array}{l}\text { Mâles adultes ..... } \\
\text { Vaches stérıles .... } \\
\text { Vaches hors d'âge. } \\
\text { Taurillons ........ }\end{array}$ & $\begin{array}{r}170000 \\
35.000 \\
182.000 \\
134.000\end{array}$ & $\begin{array}{r}28.000 \\
4.700 \\
17700 \\
8900\end{array}$ & $\begin{array}{c}15.000 \\
- \\
10.000\end{array}$ & $\frac{2.550}{-}$ & $\begin{array}{r}185.000 \\
35.000 \\
182.000 \\
144.000\end{array}$ & $\begin{array}{r}30.550 \\
4.700 \\
17.700 \\
9.600\end{array}$ & $\begin{array}{r}39.000 \\
20.000 \\
182000 \\
134000\end{array}$ & $\begin{array}{r}6.330 \\
2.600 \\
17.700 \\
8.900\end{array}$ & $\begin{array}{c}146.000 \\
15000 \\
- \\
10.000\end{array}$ & $\begin{array}{r}24.220 \\
2100 \\
\frac{7}{700}\end{array}$ \\
\hline Total des Ressources & 521.000 & 59300 & 25.000 & 3.250 & 546.000 & 62550 & $\overline{375000}$ & 35.530 & 171.000 & 27.020 \\
\hline
\end{tabular}

(1) A partir d'un cheptel total de 3.780000 Zébus et 520.000 Taurins.

ble en valeur (niveau producteur, valeur intermédiaire et valeur finale).

Malgré ses imprécisions ce tableau permet, à notre $a v \mid s$, de tirer trois conclusions essentielles :

1) Contrairement à ce que l'on a affirmé trop longtemps, le cheptel africain est explorté, il l'est même souvent de façon excessıve, surtout en ce qui concerne les jeunes animaux. Le taux d'explotation est faible, de l'ordre de 10 à 12 p. 100 des effectıfs et la part commercialisée l'est encore plus, car l'autoconsommation et l'approvisionnement des petits marchés ne provoquent aucun phénomène commercial notable. L'observateur non averti, voyant un cheptel numérıquement important, ignorant sa faible productivité et ne relevant, à première vue, qu'une commercialısation faıble, peut conclure hâtivement à la thésaurisation et à la non-exploitation du troupeau. 
2) La conversion des données numériques en données pondérales permet de constater que la production de viande est encore plus falble que ce que les taux d'exploitation lassaient supposer. Cela tient à la faiblesse des poids moyens enregistrés, partıculı̀rement pour les jeunes animaux et les vaches hors d'âge. Nous estimons que la productivité de l'élevage ofricain, exprimée en poids de viande carcosse produrfe annuellement por tête entretenue dans le troupeau est de l'ordre de $15 \mathrm{~kg}$ pour l'élevage du zébu et de $10 \mathrm{~kg}$ pour l'élevage fourın, contre $60 \mathrm{~d} 80 \mathrm{~kg}$ pour les élevages bovins européens ou américains.

Les principaux facteurs responsables de cette faible productivité sont un médiocre taux de fécondité ( 60 à 66 p. 100), une mortalıté extrêmement élevée chez les anımaux de moins de un an (30 d̀ 40 p. 100 des naissances) et enfin le manque de précocité des races locales ( 1 ) faut cinq ans pour «finir» un animal de boucherie).

3) Les animaux à vocation bouchère, issus des élevages africains, servent solt d̀ l'autoconsommation des populations rurales, soit à l'alımentation du circuit intérieur (ravitallement des petits centres et des grandes villes), soit aux exportations, qu'elles se fassent sous forme de bétail ou de viande.

En prenant l'exemple du Mali, on peut indiquer que les ressources totales se partagent de la façon suivante :

- part autoconsommée :

28.270 tonnes, soit 45 p. 100 de la production

- part commercialisée sur le marché local :

7.260 tonnes, salt 12 p. 100 de la production

- part exportée :

27.020 tonnes, soit 43 p. 100 de la production.

Sı l'autoconsommation est encore importante, constatons qu'elle est déjà ınférieure à la moitıé du disponible total.

II y a seulement vingt ans l'autoconsommation devait absorber 75 p. 100 de la production. aujourd'hul le commerce porte sur 55 p. 100 des animaux utilisables et cette part augmente régulièrement. Nous estimons qu'en 1975 le circuit commercial intéressera 62 p. 100 de la production totale du pays.

\section{II. - LES MODALITÉS DU COMMERCE DE BÉTAIL}

\section{Les achats.}

Les commerçants travaillant sur les grands circuits de bétail sont en général de gros marchands exportateurs, assez peu nombreux, mals utılisant tous plusieurs employés.

Ce commerce est également exercé par des gens dont l'actıvité se lımite à l'approvisionnement de centres locaux et d'autres qui se spécialisent dans des circuits limıtés d'un point de rassemblement à un marché de consommation.

Le véritable marchand exportateur se déplace rarement lui-même pour effectuer les achats, il charge ses employés de le faire en leur fournissant les fonds nécessaires. Cet acheteur engagera le personnel pour grouper et convoyer les animaux, il sera responsable jusqu'au stade terminal de la bonne marche des opérations.

Les achats peuvent s'effectuer sur des marchés réguliers ma1s aussı, très fréquemment, par une longue prospection dans les campements. Les éleveurs préfèrent souvent cette solution, car ils se trouvent alors en position de force face à l'acheteur. Nous avons pu constater, au cours de nos missions, qu'à de rares exceptions près les parements aux éleveurs étaient fatts au comptant.

Compte tenu de la dispersion des élevages dans toutes ces régıons, la durée des achats peut être très longue, il faut parfols plus de deux mois pour rassembler cinquante têtes de bétaıl.

\section{Les transports.}

L'acheminement a pieds. Une grande partie des déplacements d'anımaux est encore effectuée à pieds, parfois sur de très grandes distances.

Les troupeaux sont composés de 20 à 100 têtes de bovins selon les circuits étudiés et parcourent des étapes journalières de l'ordre de 25 à $30 \mathrm{~km}$. Les itinéraıres suivis sont très précis, immuables et bien connus des convoyeurs. Ils sont choisis en fonction des facilités d'abreuvement et de pâturage et évitent dans la mesure du possible les grandes zones de cultures. Les éléments des coûts de ce mode de déplacements comprennent les taxes diverses prélevées par les autorités, le salaıre des bergers (souvent forfaıtaıre), auquel il convient d'ajouter une prime pour leur nourriture ef souvent le prix de leur voyage de retour. 
Des dépenses supplémentaires peuvent inclure des redevances coutumières pour l'abreuvage du troupeau, le passage de bacs, le transit par certains marchés, aunsi que les indemnisations à payer à des agriculteurs en cas de dégâts aux cultures.

Ce mode de transport est souvent accusé de provoquer des pertes numériques et pondérables importantes, durant le parcours (1). II faut d'abord bien préciser que, pour de nombreux circuits, ces pertes sont mınimes et souvent inexistantes, parfois même les anımaux prennent du poids durant le trajet (c'est le cas en saison favorable, lorsque l'itinéraire ne sort pas de la zone sahélo-soudanienne). Sur les parcours difficiles (manque de pâturages, zones soudanoguinéennes, et forêts infestées de glossines), les pertes peuvent être conséquentes, mais sur le plan économique elles ne doivent être consıdérées que comme un des éléments du coût de transport; elles correspondent, en fait, à l'énergle dépensée par l'anımal pour se rendre d'un bout à l'autre de la chaîne de commercialısatıon. On ne peut les supprimer partiellement qu'en utilisant (lorsque cela est matériellement possible) des mayens de transport dont le coût doit être ajouté aux autres frals commerciaux.

Economiquement, l'opération n'est acceptable que lorsque cette dépense n'est pas supérieure au prix des déplacements à pieds comprenant les pertes numériques ef pondérables. En analysant la tatalıté des charges du circuit traditionnel, il est possible de calculer le coût « du transport à pieds» rapporté à la tonne de bétall vif-kilomètre. Selon les axes considérés le montant des dépenses monétalres est compris entre 2 et 6 F. CFA la tonne, bétaıl $\mathrm{vlf} / \mathrm{km}$; si l'on ajoute la valeur des pertes on peut, dans certains cas défavorables, arriver à des coûts allant jusqu'à 12 ou 14 F. CFA. Par contre, dans nombre de cas l'augmentation est lımitée à 1 ou 2 F. CFA la tonne $/ \mathrm{km}$.

Ce calcul théorıque n'aurait pas grande signification s'il ne trouvait confirmation dans les faits. Or, nous avons pu constater, que partout ou cela étaıt possible, les marchands de bestiaux utilisent

(1) On évoque également les pertes de «qualité », c'est parfois exact par rapport à des critères empruntés à la boucherie européenne, mals cela perd souvent toute signification lorsqu'on se place dans le cadre de la boucherie africaine traditionnelle. les moyens de transports modernes mis à leur disposition lorsque les conditions du milieu sont tellement éprouvantes pour le cheptel que les pertes conduisent à estimer le coût du déplacement à pieds à plus de 12 F. CFA la tonne bétail vif $/ \mathrm{km}$, ou lorsque le moyen de transport de substitution est particulièrement bon marché.

Enfin, parmi les avantages du convoyage à pieds, Il ne faut pas oublier premièrement qu'il s'agit d'un transport « porte à porte $»$, sans frais intermédiaires de chargement, déchargements, etc..., deuxièmement que c'est bien le seul moyen de transport qui «s'équilibre » tout seul en ne nécessitant aucun fret de retour. Ces éléments sont rarement pris en compte lorsqu'on essaye de comparer les qualıtés et les défauts des différents systèmes.

Les transports par voie ferrée. En Afrique de l'Ouest, les trajets ferrés: Ouagadougou-Bobodioulasso-Abidjan, Kano-Lagos, NkongsambaDovala, Yaoundé-Dovala, et à Madagascar : Tananarive-Tamatave, Antsirabé-Tamatave, Fıanarantsoa-Manakara, sont largement utilisés pour le transport du bétall.

Le prix de transport entre la Haute Volta et Abidjan est de l'ordre de $7 \mathrm{~F}$. la tonne de bétail $v i f / k m$ et l'on peut constater aujourd'hui que la grande majorité des bovins nécessaires àl'approvisionnement de la capitale de la Côte-d'lvorre, y arrive par cette voie, les autres étant acheminés par camions.

A Madagascar, entre Tananarive ef Tamatave, le prix du chemın de fer a été augmenté, portant la tonne bétail vif $/ \mathrm{km}$ à plus de $11 \mathrm{~F}$. CFA ; cela a provoqué une balsse du trafic pour le bétaıl. On constate d'ailleurs sur cet axe que les animaux de boucherie, dont la valeur finale dépend de leur embonpoint, sont toujours achemınés par wagons, alors que les taurillons de travall destınés aux paysans, dont le poids importe peu pour fixer leur valeur chez le fermier, sont convoyés d̀ pieds.

Ces exemples montrent bien que les marchands savent parfaitement utiliser au mieux les moyens de transports économiques.

Les transports par camions. L'utilisation du camion pour le transport du bétail est onéreuse. En effet, les bovins ne permettent pas d'assurer un chargement à pleine capacité des véhicules classiques. Un camion de cinq tonnes de charge utile ne peut guère transporter que huit gros 
bœufs ou dix petits, solt un pords maxımum de 2,8 tonnes. Si le tarif routier normal est de l'ordre par exemple de $10 \mathrm{~F}$. CFA la tonne $/ \mathrm{km}$, cela correspondrait pour le chargement en bétall à applıquer au moins 18 F. CFA par tonne bétaıl $\mathrm{vif} / \mathrm{km}$.

Même dans le cas de l'emplar d'un matériel routier mieux adapté, semı-remorque de vaste surface, camıon châssis long avec remorque, le coût reste très élevé, de l'ordre de 12 à 14 F. CFA la tonne, $\mathrm{km}$ bétall vif. Aussi, dans les zones fovorables ou bétall, même s'il existe un bon réseau routier, est-il antı-économıque d'envisager la substitution du convoyage traditionnel par le camionnage, ce dernier mode de transport ayant un coût moyen au moins trols fols plus élevé que les déplacements à pieds.

Cependant, dans certaines circonstances l'utılisation des véhicules se révèle possible : entre autre lorsque les transporteurs n'ont aucun fret de retour, sur des axes fréquentés par du bétal et traversant des régions défavorables, on constate que les camionneurs proposent des prix d'acheminement inférieurs au coût réel du fret.

C'est ce qui s'est passé au Ghana, où les pertes occasionnées par les déplacements sur pieds étaient consıdérables (elles pouvalent atteındre le quart du poids vif du troupeau), le réseau routier étant particulièrement dense, et les flux de transport déséquilıbrés, les camıonneurs recherchaient du fref de refour même d bas prix.

Aujourd'hui on constate que la presque totalité des animaux destinés aux centres de consommation du Sud Ghana est achemınée par camion.

Entre Fort Archambault (Tchad) et Bangui (République Centrafricaine) durant la saison des pluies, les conditions de déplacement à pieds sont extrêmement difficiles, amenant certains marchands à utiliser les transports routıers. Ils chargent des véhicules de 12 tonnes avec remorques de 5 tonnes, en y mettant 30 bceufs pour un prix global de $60.000 \mathrm{~F}$. CFA. Cette dépense, pour une distance de $634 \mathrm{~km}$, correspond à un prix de 10,5 F. CFA la tonne bétaıl vif, transportée sur un kilomètre. Cette charge est acceptable en saison des piules, mais durant la salson sèche elle n'est plus concurrentıelle avec le coût du voyage à pieds et les marchands ne l'utilısent plus.

Dans ce cas, le prix consentı par le transporteur, dont le camion a une capacité réelle de
17 tonnes, ne correspond qu'à 5,6 F. CFA par tonne $\mathrm{km}$ roulée, ce qui est très inférieur au coût réel du ircnsport.

Pour amélıorer la rentabilité de ces transports Il faudra, lorsque les conditions du milieu l'imposeroni, envisager l'emploı de camıons spécialisés, adaptés au transport des anımaux (les bétaillères), qui, permettant une utılisation à pleıne capacıté de charge des véhicules, abalssent aussi sensiblement le coût du fret.

\section{La vente des animaux dans les centres de consommation.}

C'est à ce stade du commerce que se trouvent les problèmes les plus graves.

Nous avons partout constaté que les bouchers n'achètent jamais au comptant les anımaux aux marchands de bestıcux et que ceux-ci sont obligés d'attendre plusieurs semaines, vorre plusieurs mols, pour entrer dans leurs fonds.

Nous ne traiterons pas Icl du problème de la boucherie en Afrique, mais nous devons indiquer que le plus grand désordre règne en ce domaine, dons toutes les grandes villes.

Les bouchers africaıns travallent dans les cıtés modernes, exactement dans les mêmes conditions que dans les petits villages ou sur les marchés de brousse.

L'absence de structures professionnelles, la pléthore de commerçants ımpécunieux, l'utilısation d'équipements modernes ou anciens, avec des méthodes traditionnelles souvent coûteuses, l'absence presque totale de moyens de stockage sous-froid, et la médiocrité des lieux de vente, conduisent à des gaspillages importants. Les dettes, les palabres, la lenteur des transactions et les pertes qui découlent partout de cet état de faıt, sont les travers les plus critıquables de l'ensemble du circuit commercial du bétal en Afrique. Le coût du crédıt consentı par les marchands aux bouchers, les dépenses supportées pendont l'atterte par ces marchands, la lenteur de rotation du capital, provoquent des charges importantes et stériles.

Il faut ajouter que, le plus souvent, les achats de bétall sont effectués sans que la qualité des anımaux influe considérablement sur le choix des bouchers et les prix qu'ils consentent. Fréquemment même les anımaux sont vendus par lots disparates, commerçants en bétall et bouchers se mettant d'accord sur un prix moyen par tête. 
Ces habıtudes sont directement hées aux conditions de ventes à crédit et à la nécessıté pour le vendeur de placer aussı bien les beaux animaux que les mauvais. Par la méthode de vente par lots, il arrive ainsi à se débarrasser de bêtes qui, faute d'un cours différent parfaitement défini, ne trouveraient pas preneur sur le marché.

Alors que sur des marchés parfaltement organısés Il n'est pas d'exemple que toutes les catégories de bétail, à des prix fort différents évidem. ment, ne puissent se vendre.

\section{Etude économique du commerce de bétail.}

Nous donnerons un exemple de déplacement à pieds de bétall au Camcroun, de la région d'élevage de l'Adamaoua vers Yaoundé. La distance parcourue est de l'ordre de $550 \mathrm{~km}$, la durée du parcours est de l'ordre de 20 jours, mals les délais d'attente à un poste de contrôle administratıf ef dans la capitale, pour attendre les paiements, font que l'ensemble des opérations, depuis l'achat jusqu'au retour dans la zone

TABLEAU No 4

Frais d'acheminement à pieds et de commercialsation du bétoil au Cameroun, de la zone d'élevage de l'Adomaoua à Yooundé

Unités : F. CFA pourcentages.

\begin{tabular}{|c|c|c|c|c|}
\hline \multirow{2}{*}{ Postes de dépenses } & \multirow{2}{*}{$\begin{array}{l}\text { Pour un troupeau } \\
\text { de } 40 \text { rêtes }\end{array}$} & \multicolumn{2}{|c|}{ Par tête } & \multirow{2}{*}{$\frac{\text { Remarques }}{\text { p } 100 \text { de la valeur finale }}$} \\
\hline & & en valeur & p. 100 & \\
\hline 1) Achat animal $400 \mathrm{~kg}$ vif (1).... & 588000 & 14.700 & 73,5 & \\
\hline $\begin{array}{l}\text { I1) Taxes de marché, d'exportation, } \\
\text { sanitaire et patente du mar- } \\
\text { chand } \ldots \ldots \ldots \ldots \ldots \ldots \ldots \ldots \ldots\end{array}$ & 20.000 & 500 & 2,5 & \multirow[b]{2}{*}{ Zone de prospection facile. } \\
\hline $\begin{array}{l}\text { III) Frass de collecte et de rassem- } \\
\text { blement } \ldots \ldots \ldots \ldots \ldots \ldots \ldots\end{array}$ & 4.000 & 100 & 0,5 & \\
\hline 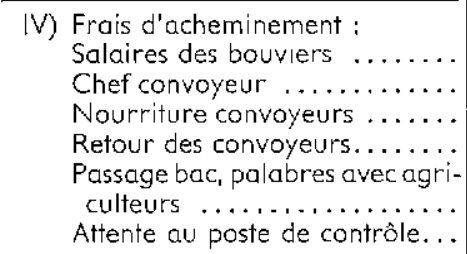 & $\begin{array}{r}14.000 \\
14.000 \\
15.000 \\
2.500 \\
10.000 \\
6000\end{array}$ & $\begin{array}{r}350 \\
350 \\
375 \\
62 \\
250 \\
150\end{array}$ & & \multirow{3}{*}{$\begin{array}{l}\text { Le coût du déplacement y } \\
\text { compris les pertes est d'env. } \\
2900 \mathrm{~F} / \mathrm{CFA} \text { par tête Pour } \\
\text { un bouf arrivant de Yaoun- } \\
\text { dé au poids de } 380 \mathrm{~kg} \text { et } \\
\text { ayant parcouru } 550 \mathrm{~km} \text {, le } \\
\text { coût du transport est d'env. } \\
13 \mathrm{~F} / \mathrm{CFA} \text { la tonne/km. Sans } \\
\text { compter les perles la dépen- } \\
\text { se est de 7F/CFAlatonne } / \mathrm{km} \text {. }\end{array}$} \\
\hline $\begin{array}{l}\text { Total des frais } \ldots \ldots \ldots \ldots \ldots \ldots \\
\text { Pertes totales } 0,3 \ldots \ldots \ldots \ldots \ldots \\
\text { Pertes poids } \text { if } 5 \text { p. } 100 \ldots \ldots \ldots \\
\text { Manque d̀ gagner par ventes } \\
\text { d'urgence } \ldots \ldots \ldots \ldots \ldots \ldots\end{array}$ & $\begin{array}{r}61.500 \\
240 \\
40000 \\
10600\end{array}$ & $\begin{array}{r}1.537 \\
60 \\
1.000 \\
265\end{array}$ & 7,7 & \\
\hline Total des pertes............ & 50.840 & 1.325 & 6,6 & \\
\hline \multirow[t]{2}{*}{$\begin{array}{l}\text { v) Frais généraux du marchand. } \\
\text { Voyage } \ldots \ldots \ldots \ldots \ldots \ldots \ldots \ldots \\
\text { Frais de séjour Yaoundé...... } \\
\text { Frais financiers } \ldots \ldots \ldots \ldots \ldots\end{array}$} & $\begin{array}{r}4.000 \\
12.000 \\
16.000\end{array}$ & $\begin{array}{l}100 \\
300 \\
400\end{array}$ & & \\
\hline & 32.000 & 800 & 4 & \\
\hline VI) Bénéfice du marchand......... & 40.000 & 1.000 & 5 & \\
\hline $\begin{array}{l}\text { VII) Valeur finale sur le marché, } \\
\text { chiffres arrondis ............. }\end{array}$ & 800.000 & 20.000 & & \\
\hline
\end{tabular}

(1) Les animaux pèsent $400 \mathrm{~kg}$ au départ et $380 \mathrm{~kg}$ à l'arrivée (hypothèse perte $5 \mathrm{p} .100$ ). 
d'élevage, demandent de deux ò trois mois, délars qui seralent réduits de moitié si le parement au comptant étalt effectué à Yooundé.

Le tableau No 4 donne le détall des dépenses à retenir pour le transport, les hypothèses appltquées pour les pertes sont pessimistes, afin de ne pas minimiser les charges.

Pour permettre de comparer deux méthodes d'acheminement, nous présentons au tableau No 5 les dépenses représentées par la commercılisation sur le même axe «Adamaoua-Yaoundé» d'un troupeau de 10 têtes transporté par camions. Nous considérerons que les pertes sont nulles et les frais généraux du marchand plus faibles que dans le premıer exemple.

Malgré la suppression des pertes de poids et la réduction des frals généraux, le prix du bétal n'a pas été amélioré dans la zone de consommation. En effet, le prix du kilogramme vif de l'animal, transporté par camıon, est de 55,9 F.
CFA et celui de l'animal venu à pied de 52,6 F. CFA.

L'économı du système moderne réside dans la sauvegarde de $20 \mathrm{~kg}$ de poids vif par animal transporté ; cette économie de substance noble est forf importante; elle ne permet cependant pas, comme certans programmes d'amélioration le proposent, de poyer mieux le producteur ou d'abaisser le prix de la viande dans le lieu de consommafion.

\section{III. - LA DEMANDE}

L'autoconsommation rurale et la demande des petits centres et des marchés situés dans les zones d'élevage sont loin d'être néglıgeables et s'accroissent régulièrement. Cependant, nous avons constaté que la partie commercialisée devenait de plus en plus importante et augmentalt plus rapidement que la production elle-même.

TABLEAU No 5

Frois de transport par camion et de commercialisation du bétall au Comeroun de la zone d'élevage de l'Adamaoua à Yaundé

Unité : F. CFA.

\begin{tabular}{|c|c|c|c|c|}
\hline \multirow{2}{*}{ Postes de dépense } & \multirow{2}{*}{ Pour 10 têtes } & \multicolumn{2}{|c|}{ Par tête } & \multirow{2}{*}{$\frac{\text { Remarques }}{\text { p. } 100 \text { de la valeur finale }}$} \\
\hline & & en valeur & P 100 & \\
\hline 1) Achat anımal, $400 \mathrm{~kg}$ vif...... & 147.000 & 14700 & 65,6 & \\
\hline $\begin{array}{l}\text { II) Taxe marché, exportation et } \\
\text { patente du marchond......... }\end{array}$ & 4.000 & 400 & 1,8 & $\begin{array}{l}\text { Le voyage camionéconomise } \\
\text { la prévention (pas de taxe } \\
\text { sanitaire) }\end{array}$ \\
\hline $\begin{array}{l}\text { III) Frais de collecte et de rassem- } \\
\text { blement } \ldots \ldots \ldots \ldots \ldots \ldots \ldots\end{array}$ & 1.000 & 100 & 0,4 & \\
\hline $\begin{array}{l}\text { IV) Transport camion : } \\
\text { Frais de chargement } \ldots . . . \ldots \\
\text { Prix du transport............ } \\
\text { Salaire et transp convoyeur... } \\
\text { Dimes et frais divers......... } \\
\text { Déchargement } \ldots \ldots \ldots \ldots \ldots\end{array}$ & $\begin{array}{r}4.000 \\
45000 \\
6500 \\
500 \\
500\end{array}$ & $\begin{array}{r}400 \\
4.500 \\
650 \\
50 \\
50\end{array}$ & & $\begin{array}{l}\text { La route est plus longue que } \\
\text { le chemin direct : } 900 \mathrm{~km} \text { au } \\
\text { lieu de } 550 \text {, le caût du trans- } \\
\text { part représente cependant } \\
\text { encore } 17 \mathrm{~F} \text {. CFA la tonne } / \mathrm{km}\end{array}$ \\
\hline & 56500 & 5.650 & 25,2 & \\
\hline $\begin{array}{l}\text { V) Frais généraux du marchand : } \\
\text { Voyage marchand } \ldots \ldots \ldots \ldots \ldots \\
\text { Frais de séjour } \ldots \ldots \ldots \ldots \ldots \\
\text { Frais financiers } \ldots \ldots \ldots \ldots \ldots\end{array}$ & $\begin{array}{l}1.000 \\
2500 \\
1.750\end{array}$ & $\begin{array}{l}100 \\
250 \\
175\end{array}$ & & \\
\hline & 5.250 & 525 & 2,5 & $\vdots$ \\
\hline VI) Bénéfice du marchand........ & 10.000 & 1.000 & 4,5 & . \\
\hline VII) Valeur finale sur le marché.... & 223.750 & 22.375 & & ' \\
\hline
\end{tabular}


TABLEAU Na 6

Evolution de ta demande de viande dans différentes capitales africaines et malgoches (1954-1965)

Unıtés : tonnes de produits ou nombre de bovins abattus.

\begin{tabular}{|c|c|c|c|c|c|c|}
\hline \multirow{2}{*}{ Années } & Abıdjan & Tananarive & Banguı & Fort-Lamy (1) & Bamako & Accra \\
\hline & $\begin{array}{l}\text { ronnages toutes } \\
\text { viandes ef abats }\end{array}$ & \multicolumn{3}{|c|}{$\begin{array}{l}\text { tonnages toutes viandes } \\
\text { (abats non compris) }\end{array}$} & \multicolumn{2}{|c|}{$\begin{array}{c}\text { nombre de bovins } \\
\text { abatlus }\end{array}$} \\
\hline $\begin{array}{l}1954 \ldots \\
1955 \ldots \\
1956 \ldots \\
1957 \ldots \\
1958 \ldots \\
1959 \ldots \\
1960 \ldots \\
1961 \ldots \\
1962 \ldots \\
1963 \ldots \\
1964 \ldots \\
1965 \ldots\end{array}$ & $\begin{array}{l}3.092 \\
3.915 \\
3.906 \\
4.330 \\
4421 \\
3.926 \\
4.115 \\
4.993 \\
5498 \\
6525 \\
8.095 \\
-\end{array}$ & $\begin{array}{r}7.192 \\
7.960 \\
8.814 \\
8.829 \\
9.464 \\
10.054 \\
9.970 \\
10.443 \\
11.692 \\
- \\
-\end{array}$ & $\begin{array}{l}1538 \\
1.522 \\
1.723 \\
1.839 \\
1.824 \\
2.059 \\
2.490 \\
2664 \\
2.582 \\
3.047 \\
3.263 \\
3665\end{array}$ & $\begin{array}{c}- \\
- \\
- \\
- \\
1.685 \\
2.096 \\
2.295 \\
2625 \\
2.767 \\
3.263 \\
3.264\end{array}$ & $\begin{array}{c}18.371 \\
19.531 \\
21007 \\
21.666 \\
21.134 \\
23.345 \\
- \\
27.781 \\
- \\
31602 \\
35.520 \\
-\end{array}$ & $\begin{array}{c}- \\
- \\
- \\
11.070 \\
11.892 \\
15.030 \\
16.434 \\
20.524 \\
21.710 \\
27.197 \\
-\end{array}$ \\
\hline $\begin{array}{l}\text { Taux an- } \\
\text { nuel d'ac- } \\
\text { croisse- } \\
\text { ment }\end{array}$ & $\begin{array}{c}10,1 \text { p. } 100 \mathrm{de} \\
1954 \text { d̀ } 1964\end{array}$ & $\begin{array}{l}6,2 \text { p. } 100 \text { de } \\
1954 \text { d̀ } 1962\end{array}$ & $\begin{array}{l}8,2 \text { p. } 100 \text { de } \\
1954 \text { d̀ } 1965\end{array}$ & $\begin{array}{l}11,4 \text { p. } 100 \mathrm{de} \\
1959 \text { à } 1965\end{array}$ & $\begin{array}{l}6,8 \mathrm{p} \cdot 100 \mathrm{de} \\
1954 \text { d̀ } 1964\end{array}$ & $\begin{array}{l}16 \mathrm{p} 100 \mathrm{de} \\
1958 \text { d̀ } 1964\end{array}$ \\
\hline
\end{tabular}

(1) Exportalions non comprises.

\section{La demande globale.}

La demande des grandes villes et notamment des capitales s'accroît en effet de façon considérable ainsi d'ailleurs que la consommation globale des pays insuffisamment pourvus en bétail.

Le tableau No 6 donne quelques exemples de développement récent de l'approvisionnement en vande (abattue localement ou importée) dans différentes capıtales africaınes et malgaches.

On constate un développement spectaculare de la consommation globale de ces villes, à un taux annuel moyen dépassant partout 6 p. 100 et parfoss égal ou supérieur à 10 p. 100. Cette évalution très marquante dans les grandes capitales, se retrouve également dans les centres de petıte et moyenne importance. En Côted'Ivoire, par exemple, les abattages des villes autres qu'Abidjan ont augmenté de 130 p. 100 en 11 ans, soit à un taux moyen annuel proche de 8 p. 100.

\section{La consommation individuelle.}

Le niveau de consommation individuelle atteint dans les grandes villes est en général élevé, ainsi qu'en témoigne le tableau suivant:

\section{TABLEAU No 7}

Nweau de consommation annuelle par habitant dons quelques grandes villes africanes et malgaches

Unité : kılogramme de viande et d'abats.

\begin{tabular}{|c|c|c|c|}
\hline Centres & $\left|\begin{array}{c}\text { Année } \\
\text { consi- } \\
\text { déréé }\end{array}\right|$ & $\begin{array}{l}\text { Consom- } \\
\text { mation } \\
\text { Indivi- } \\
\text { duelle } \\
\text { moyenne }\end{array}$ & Observations \\
\hline $\begin{array}{l}\text { Lomé } \ldots . \\
\text { Accra .... } \\
\text { Douala ... } \\
\text { Dakar ... } \\
\text { Abidjan ... }\end{array}$ & $\begin{array}{l}1963 \\
1960 \\
1963 \\
1962 \\
1964\end{array}$ & $\begin{array}{l}13,6 \\
14,0 \\
18,6 \\
20,1 \\
27\end{array}$ & $\begin{array}{l}\text { Pour toutes ces villes la } \\
\text { consommation de vian- } \\
\text { de et d'abats est com- } \\
\text { plétée par une très } \\
\text { forte consommation de } \\
\text { poisson comprise entre } \\
25 \text { et } 50 \mathrm{~kg} \text { par habi- } \\
\text { tant et par an. }\end{array}$ \\
\hline Bangui ... & 1965 & 32,9 & \\
\hline $\begin{array}{l}\text { Bamako.. } \\
\text { Tananarive } \\
\text { Fort-Lamy } \\
\text { Nouakchott }\end{array}$ & $\begin{array}{l}1964 \\
1962 \\
1965 \\
1961\end{array}$ & $\begin{array}{l}37,7 \\
43,8 \\
50 \\
72,5\end{array}$ & $\begin{array}{l}\text { Villes proches des zones } \\
\text { de production dispo- } \\
\text { sant d'un approvision- } \\
\text { nement facile abon- } \\
\text { dant et bon marché. }\end{array}$ \\
\hline
\end{tabular}


Contrairement à des opinions fréquentes, mals mal fondées, le niveau moyen des consommations indıviduelles urbaines, est réellement élevé dans la majorité des grandes capıtales. Assortı d'une croissance démographique accélérée, ce facteur provoque la véritable explosion de la demande que nous avons constatée précédemment.

Devant une teile situation, Il paraît très important d'évaluer rapidement l'évolution prévisible des besoins au cours des dix ou vingt prochaines années. C'est ce que naus avons tenté dans différentes études pour un certaın nombre de pays ou de villes (tableau No 8).

\section{TABLEAU No 8}

Hypothèses d'évolution prévisible des besons de certaines villes ou de certans poys africoins

Unıtés : tonnes de viande et d'abals.

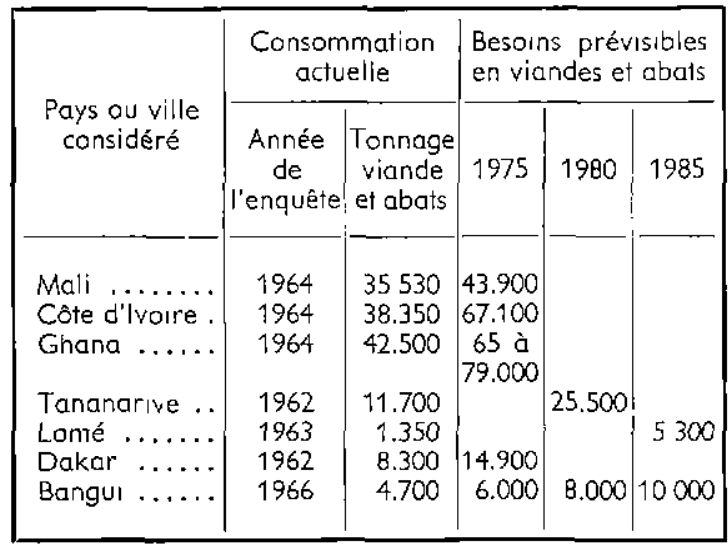

De telles prévisions sont évidemment hasardeuses et nous prions le lecteur de les considérer seulement comme des ordres de grandeur. Nous n'avons volontarrement jamaıs appliqué dans ces calculs de données trop optımistes.

Ces hypothèses, volontaırement raisonnables, correspondent cependant à des accroissements de 4 p. 100 et plus. Or, dans les conditions actuelles, on ne peut guère envisager un taux d'améliaration de la production supérieur à deux ou trols pour cent par an, à condition d'éviter toute exploitation anarchique, comme nous l'avons constaté par exemple pendant une certaine période en Adamaova (Cameroun) ou à Madagascar (en 1930 approximativement).

Dans l'état actuel de nos connalssances, on peut donc seulement remarquer qu'à très court terme l'offre aura beaucoup de mal à suivre la demande.
Sur le plan économique, il est nécessaire d'aborder le problème du prix de la viande au détail, dans les centres de consommation, et son incidence sur les prix payés au producteur.

Il faut savor que le seul prix de défail sıgnificatif est celur de la viande vendue avec os sur les marchés africains, car au morns 90 p. 100 de la distribution est réalisé sous cette forme.

Les prix actuels au $\mathrm{kg}$ de viande avec os sont approximativement les suivants pour quelques villes : Dakar de 150 à 190 F. CFA (selon la saison)-Abıdjan : 190 F. CFA - Bangul - 110 à $120 \mathrm{~F}$. CFA. Ils sont relativement faibles et souvent iis n'ont pas suivi le rythme d'augmentation de nombreux prix de prodults vivriers ou de biens de grande consommation.

A Abrdjan, par exemple, il y a stabilité du prix de la viande au détal entre 1961 et 1965, à Bangui de 1954 à 1965. On constate d'alleurs que les hausses de prix suivent en général une période de crise sur le marché du bétail : à un moment donné les autorités qui partout maintiennent des prix taxés assez serrés sont obligées, sous les pressions commerciales, de lâcher un peu les tarifs en vigueur. Le système n'est pas souple, d'abord à cause des perpétuelles discussions sur le marché du bétall et de l'ignorance de nombreux bouchers quant à leur prix de revient et à leurs marges, ensuite parce que les prix taxés ne peuvent évolver que par «tranches de 5 francs », du fait des habitudes monétaires et $d u$ manque de monnaie divisionnaire.

Nous pouvons affirmer, pour l'avoir contrôlé souvent, que les bouchers ne volent pas au poids, ce qui serait une façon d'ajuster leur prix, mais lorsque le marché leur est défavorable ils augmentent dans leurs ventes le pourcentage d'os, de déchets ou d'abats.

II nous semble, que, dans les années prochaınes comple tenu de la diversification et du fort accroissement de la demande, ces prix ne pourront pas rester aux niveaux actuels. L'exemple d'Accra est significatif, car pour diverses difficultês économiques et monétaires, et du fait de la vive concurrence de la Côte-d'ivoire, nous avons relevé en 1965 le prix de vente de la viande au détail le plus élevé : près de $400 \mathrm{~F}$. CFA par $\mathrm{kg}$ avec os et abats.

Ce prix de la viande a une incidence directe sur la valeur afferte à l'éleveur par les marchands. Notons tout d'abord que certains écono- 
mistes faisant référence aux conditions européennes considèrent que le prix payé aux éleveurs sahéliens est particulièrement faible. Il faut se garder d'un tel jugement car, à notre avis, ces comparaisons n'ont pas grand sens. En effet, à partir de son capital bétail, l'activité essentielle du pasteur africain, et surtout malgache, consiste avant tout à prélever le disponible annuel «d'une production quasispontanée» he faisant pratiquement aucune dépense intermédıare (soins, nourriture, logement, etc...). Comme pour tous les produits de cueillette, la valeur est avant tout fonction de l'offre ef de la demande alors que pour un produit élaboré le coôt des facteurs de production et des matières premières en fixera le prix plancher.

Aussi pour le cheptel africain c'est la richesse, l'éloignement et les quantités nécessaires à l'approvisionnement des marchés à servir qui détermineront le niveau moyen des prix à la production. Pour cette raison, certains types d'anımaux sont nettement mieux payés que d'autres, non en fonction de leur qualité intrinsèque, mais uniquement en fonction de leur capacité d'atteindre à pied, dans de bonnes conditions, cerraıns marchés éloignés.

En Afrique de l'Ouest, pour ces raisons, on relève des disparités considérables : par exemple, le même type d'animal d'exportation se paye environ $25 \mathrm{~F}$. CFA le $\mathrm{kg}$ vif dans l'Est du Tchad et 50 F. CFA au Sénégal ou au Malı.

Le premier sera revendu sur des marchés distants de 1.500 à $3.000 \mathrm{~km}$ (Bangui ou Lagos), alors que le second approvisionnera Dakar ou Abidjan, beoucoup moins élorgnés et plus faciles d'accès à partir de la zone de production.

\section{PERSPECTIVES ET CONCLUSIONS}

\section{«La production doit être développée »}

Parmi les considérations d'ensemble que nous venons de présenter sur le commerce du bétall en Afrique de l'Ouest et à Madagascar, l'une d'entre elles $a$, croyons-nous, une importance plus grande; Il s'agit du déficit important en viande de boucherie que nous sommes amenés à prévoir pour les vingt prochaınes années.

L'élevage africain est actuellement largement exploıté, la commercialısatıon traditionnelle est de plus en plus active; dans certaines zones des formules nouvelles prennent progressivement le relals. Si l'on veut éviter que très rapıdement le cheptel soit menacé par une sur-exploitation, il faut absolument que tous les programmes de développement accordent une ploce prioritare aux actions tendant à augmenter la production de viande et à rationalıser sa transformation et sa distribution.

Contrairement à d'autres produits agricoles africaıns, dont le placement est parfois difficile, la production animale est assurée de trouver un débouché en Afrique même et à un prix de plus en plus rémunérateur.

Dans les pays guinéens et soudano-guinéens, on dolt tout mettre en œuvre pour améliorer les élevages sédentaires de bovins trypano-tolérants et intégrer les animaux aux activités terriennes dans le cadre de la modernisation des techniques agricoles. Dans ces régions, la mise en place d'élevages de porcs, de petits ruminants et de volalles doit être poursuivie sans relâche pour accroître le disponible en protéines aussi bien chez les ruraux que dans les villes.

L'élévation de la productivité du cheptel extensif sahélıen pose des problèmes extrêmement complexes à résoudre : pathologie, protection des jeunes, hydraulique pastorale et surtout évolution des méthodes d'élevage de ces populations transhumantes très difficiles à aborder.

Cependant, on peut aussi envisager d'améliorer la quantité de viande produite en essayant d'ajouter du poids aux animaux issus de ces élevages extensifs par des techniques plus modernes.

De l'élevage traditionnel, considéré comme «naisseur», les animaux, surtout les taurllons, pourralent passer à un stade d'《embouche» soit sur herbages naturels : chez des éleveurs spécialısés (comme dans le moyen-ovest malgache) ou dans des ranches, soit par des actions plus modernes, combinant la production de fourrages à haut rendement et l'utilisation de sous-produits de l'industrie alimentare aujourd'hun souvent gaspillés (ou exportés vers des pays riches). Ces dernières méthodes demandent d'alleurs de très sérleuses études économiques avant d'être proposées et diffusées sur une grande échelle. 


\section{Les améliorations souhaitables dans le domaine commercial :}

Pour que ces efforts de production solent encouragés et rentabılisés il est indispensable que les modalités commercıales soient amélıorées. Et contraırement à ce qu'une analyse superficielle pourralt lasser supposer, c'est d'abord dans les centres de consommation qu'il faut intervenir ovec le plus de vigueur.

Il est nécessaire d'abord de mettre en place des équipements bien adaptés aux besoins et convenablement gérés :

- marchés à bétaıl, sımplement maıs commodément aménagés,

- abattoirs-frigorifiques modernes, permettant une transformation hygiénique et économique de la viande et une récupération aussı complète que possible des sous-produits,

- marchés de gros de la viande, indispensables pour la mise en concurrence des circults vifs et forains,

- marchés de détall, offrant des bonnes conditions de distribution, avec au moins, pour les principaux d'entre eux, de modestes possibilités de stockage sous froid pour les invendus.

Un équipement satisfaisant, utılisé ratıonnellement, est indispensable pour amélıorer la commercialısation, mais il sero inopérant si conjorntement on ne met pos f'ordre dons les professions intéressées : bouchers grossistes, détaillants, courtiers en bétall, etc... II faut, par exemple, rendre obligatoire, à plus ou moins long terme, les poiements au comptant et la détermination de prix établis selon, ou mons, deux qualités, si possible après pesée des anımaux.

Les modalités des transactions doivent être suivies avec attention par des services compétents, dont les contrôles seron1 de tross ordres : fiscaux et surveillance des prix, sanitaires et statistiques. Cela afin de suivre l'évolution du marché ef éventuellement d'intervenir en fonction de ses fluctuations.

Telles sont les mesures essentielles qui permettront ensuite d'amélıorer l'ensemble du système en remontant la chaîne commercıale jusqu'au producteur. La création des coopératives de producteurs ou de véritables circuits de viandes foraines ne peut être envisagée tant que les structures professionnelles des centres consom- mateurs resteront ce qu'elles sont, tous les échecs que nous avons constatés tiennent à ces conditions commerciales anarchiques.

Pour rendre plus rapide la collecte des animaux dans les zones de production et apporter aux éleveurs les biens de consommation dont ils ont besoin, l'ouverture de marchés réguliers est souhaltable. Mais la vie d'un marché n'est pas le fait d'une simple décisıon administrative, elle ne dépend que de sa fréquentation assidue par les marchands (de bétall, de tıssus, de produits alımentaires, etc...) et par les éleveurs, c'est-d̀dire, avant tout que des facilıtés de communication offertes. La création de routes ou de pistes, permettant aux «taxis-brousse » de passer, a souvent provoqué la nassance de marchés très actifs, alors que nous connaissons beaucoup de forrails, créés unıquement par décisıon adminıstrative sans études préalables, qui sont restés sans vie et sans activité.

A partir des marchés de regroupement, l'amélioration des transports et des déplacements est aussı fonction des progrommes d'équipements d'infrastructure et de l'abaissement des taux de fret routier ou ferroviaire.

On doit retenir pourtant que l'achemınement du bétail de la zone d'élevage, écologıquement favorable, ainsi que dans ses abords immédıats, se fera pour longtemps encore par convoyoge à pied. Cela non pas pour des raisons de résistances rétrogrades et coutumières d̀ des instruments de progrès, mais beaucoup plus simplement pour des roisons économiques. En conséquence, certaınes réalisations, ou de sımples dispositions légales, doivent permettre de poursuivre et peutêtre d'améliorer ce transport économiquement rotionnel. On songe taut naturellement au ravitallement en eau sur certains parcours semidésertiques, mass ce n'est pas le seul problème Par exemple, lorsque les grands axes de commercıalısation traversent des régions à fortes densıtés humaınes et agricoles, Il faut prévoir l'aménagement de chemınements pour les convois, en réservant légalement des parcours suffisants.

La construction de parcs, aux étapes imporantes ou obligatoires (passages forcés), peut être envisagée afin d'éviter la divagation des animaux et les dégâts aux cultures.

Enfin, sı des modalités de transports améliorés peuvent se substituer à l'avenır aux déplacements à pied pour les troupeaux destınés aux 
très grandes villes ; I est peu probable que cela soit possible pour servir la multitude de centres de moyenne importance et les régions rurales des pays demandeurs.

En effet, pour cette demande, très souvent saisonnière, correspondant aux périodes de «tralte» des grands produits agricoles, seul le commerçant, déambulant dans le pays avec son troupeau, est en mesure d'en assurer l'approvisionnement souvent avec des animaux légers, assez coûteux, qul conviennent mieux aux bouchers ef aux agriculteurs de ces régions.

Or, l'importance relative de ce marché est forte : souvent plus de la moitié des importations totales de bétall (Côte-d'lvoire, Ghana, République Centrafricane).

\section{Les circuits de viande foraine.}

Avant de conclure, et bien que ce problème sorte du cadre strict du commerce de bétail, nous examinerons rapidement la création dans un passé récent des premiers circuits africaıns de viande foraine et quel peut être leur avenir.

C'est à Fort Lamy (Tchad) que, dès 1948, des expéditions de viandes réfrigérées par avion ont été tentées et se sont développées. Depuis, de Bamako, de Niamey, de Ouagadougou et Bobodıoulasso, de nouveaux circuits ont été ouverts, les transports étant effectués soit par avion, soit par camion et wagons frigorifiques.

Pour donner une idée de l'importance prise par ce mode de commercialisation, nous prendrons l'exemple de Fort Lamy comme centre expéditeur ef de la Côte-d'ivolre, comme lieu de réception (tableau No 9).

\section{TABLEAU No 9}

Expédifions de vandes du Tchad de 1948 à 1965

Unités : Tonnes de carcasse.

\begin{tabular}{|r|r|r|r|r|c|}
\hline Année & Tonnage & Année & Tonnage & Année & Tonnage \\
1948 & 59 & 1954 & 2.745 & 1960 & 5.056 \\
\cline { 3 - 5 } 1949 & 61 & 1955 & 3.135 & 1961 & 5.186 \\
1950 & 329 & 1956 & 3.172 & 1962 & 4.155 \\
1951 & 1.048 & 1957 & 3.439 & 1963 & 4.543 \\
1952 & 2.102 & 1958 & 4.261 & 1964 & 4.433 \\
1953 & 2.075 & 1959 & 3.810 & 1965 & 4456 \\
\hline
\end{tabular}

Les marchés servis par Fort Lamy sont actuellement, par ordre d'importance, Brazzaville,
Painte Noire, Libreville, Port Gentil, Douala, Dolısıe, Yaoundé, Trıpolı, Kınshasa, etc... Tous les transports sont effectués par avion, seule solution techniquement utilisable pour l'instant.

La Côte-d'ivaire assure son approvisionnement depuls plusieurs années en important des viandes foraines en provenance d'Europe ou des différents pays africains voisıns : Mali, Niger, Haute-Volta et parfois Sénégal. Le volume de ces importations, en accroissement régulier de 1952 d̀ 1955, a ensulte peu varié jusqu'en 1960, par contre depuis 1961 l'augmentation est très importante et la proportion des viandes provenant d'Afrique atteint un niveau élevé au détrıment des importations européennes, ainsi que le montre le tableau No 10.

\section{TABLEAU No 10}

Importations de viandes foraines en Côte d'fvorre Ventilation por origine

Unıté : tonne de carcosse.

\begin{tabular}{|c|c|c|c|c|c|}
\hline \multirow{3}{*}{ Année } & \multirow{3}{*}{$\begin{array}{l}\text { Tonnage } \\
\text { total } \\
\text { importé }\end{array}$} & \multicolumn{4}{|c|}{ Ventilation par origine } \\
\hline & & \multicolumn{2}{|c|}{$\begin{array}{c}\text { Viande importée } \\
\text { d'Europe }\end{array}$} & \multicolumn{2}{|c|}{$\begin{array}{l}\text { Viande importée } \\
\text { de pays africains }\end{array}$} \\
\hline & & Tonnage & $\begin{array}{l}\text { P. } 100 \\
\text { du tolal }\end{array}$ & Tonnage & $\mid \begin{array}{l}\text { P. } 100 \\
\text { du total }\end{array}$ \\
\hline 1952 & 206 & 125 & 61 & 81 & 39 \\
\hline 1953 & 234 & 121 & 52 & 113 & 48 \\
\hline 1954 & 401 & 123 & 31 & 278 & 69 \\
\hline 1955 & 528 & 211 & 40 & 317 & 60 \\
\hline 1956 & 480 & 203 & 42 & 277 & 58 \\
\hline 1957 & 508 & 187 & 37 & 321 & 63 \\
\hline 1958 & 478 & 168 & 35 & 310 & 65 \\
\hline 1959 & 455 & 126 & 28 & 329 & 72 \\
\hline 1960 & 589 & 104 & 18 & 485 & 82 \\
\hline 1961 & 1.009 & 178 & $\uparrow 7$ & 831 & 83 \\
\hline 1962 & 1.114 & $\uparrow 48$ & 13 & 966 & 87 \\
\hline 1963 & 1.511 & 217 & 14 & 1.294 & 86 \\
\hline 1964 & 1.706 & 228 & 13 & 1.478 & 87 \\
\hline
\end{tabular}

Pour apprécier les chances de développement de ce cırcult moderne, il est nécessaire de considérer dans quelles conditıons il a atteint le niveav actuel.

La caractéristique essentielle des marchés ouverts est, qu'au départ, ils n'ont touché qu'une clientèle riche. D'abord parce que les marges commerciales étaient suffisantes, ensuite parce que l'infrastructure d'accueil existait dans les grandes villes pour recevoir ces viandes, ou a été 
créée de toutes prèces par les sociétés exportatrices elles-mêmes, ofin de servir une demande exigeonte.

La qualité des viandes offertes devalt être excellente et c'étalent surtout les morceaux de choix qui étaient demandés. Compte tenu de la rapidité des transports, de la nécessité de serrer les prix, mais aussi du goût de la clıentèle, toutes ces expéditions ont été réalisées sous forme de viandes réfrigérées (de 0 d̀ +2 degrés celsius).

L'expérience acquise aujourd'hul permet d'affirmer que pour réussir, les expéditions dolvent être faites à partir d'abattoirs frıgorıfiques bien conçus, installés non pas au cœur de la zone d'élevage, mais dans une grande ville située d proximité de celle-ci. Les ralsons de ce charx sont de trois ordres :

- l'abattoir industriel permet de servir à la fors les besoins de la ville où il est implanté, ce qui accroît sensiblement sa rentabılité. Par exemple, les besoins de Fort Lamy sont de plus de 3.200 tonnes par an et ceux de Bamako de près de 4.000 tonnes, dont les abattages correspondants assurent à ces établissements une activité de base essentielle pour leur fonctionnement.

I! faut aussi consıdérer que ces marchés locaux ont une ampleur suffisante pour mieux valoriser le cinquième quartıer et absorber des catégories de viandes qui ne sont pas exportées (pour certains marchés par exemple, on expédie Jes quartiers arrières ef l'on vend sur place les avants dans de bonnes conditions).

- La présence dans ces grandes villes d'une infrastructure commerciale et de services publics étoffés est indispensable à l'abattor et aux exportateurs de viandes. L'entretien des installations est beaucoup plus alsé que dans les zones rurales, les fournitures d'énergie, d'eau sont en général assurées et le recrutement du personnel est facilité.

- Pour les expéditions, c'esł à partır des grands centres que l'on trouve les possibilités les meilleures : routes, aérodromes, voles ferrées et services de transports réguliers.

Pour développer aujourd'hul ces méthodes modernes de service sur des marchés africaıns, f'obstacle majeur n'est plus d'orıgıne économique. En de nombreux cas, en effet, on peut, surtout sı l'on dispose d'un moyen de transport économique, proposer des viandes foraınes ne coûtant pas plus cher que les carcasses abatłues sur place.
Une fols encore, la difficulté se trouve dans l'anarchie des professıons ef l'ımpécunıosité des bouchers africans, ainsı que dans le manque d'instaliations indispensables.

Sı, à Á̉ıdjan, les viandes forcines pénètrent peu à peu sur les grands marchés, c'est qu'il existe quelques bouchers grossistes entreprenants ef que les nouvelles installations de ventes sont parfaıtement conçues, ef équipées de chambres froides permettant la conservation des quartiers. Une mise en ordre de la structure traditionnelle des bouchers, l'aménagement de l'abattoir et d'un marché de gros permettront dans cette ville d'accroitre encore plus rapidement l'ımportance du circult forain. En effet, l'objectif ne doit pas être de remplacer totalement l'approvisionnement en bétall vif par le nouveau système, mais au contrare de le développer harmonieusement en libre concurrence.

Nous sommes donc persuadés que les viandes foraines peuvent, à l'avenır, prendre une place importante sur les marchés de consommation, à condition :

- de n'envisager que des viondes réfrigérées, car les bouchers afrıcains ne peuvent pas travailler de viandes congelées et que les coûts de la congélation, des transports à $-18^{\circ} \mathrm{C}$ et de stockage à cette température sont beaucoup trop élevés pour faıre concurrence aux viandes abattues sur place.

- que les pays demandeurs fassent un sérieux effor d'organisation et d'équpement. Cet effort, bien que cela n'apparaisse peut-être pas clairement aujourd'hul, sera absolument nécessaire pour résoudre leurs problèmes d'approvisionnement. Il est actuellement navrant de constater l'état des abattoirs et des lieux de vente de la viande dans des villes comme Accra, Lagos ou Dovala.

- qu'aucune barrière, de quelque nature que ce soit. ne subsiste à l'introduction des viandes fora nes provenant de pays africains voisins. La Nigeria, par exemple, maintient une taxe de 50 p. 100 ad valorem sur les viandes qui pourralent lui être expédiées du Niger et du Tchad. Ce pays, le plus fort impartateur de toute l'Afrique de l'Ouest, bloque ainsı, sans doute pour maintenir des privilèges locaux, toute amélıoratıon commerciale à long terme. Pourtant ces amélıorations sont indispensables oux pays fournisseurs pour amélıorer leur productivité ef accroître ainsi 
une production dont la Nigerı aura inéluctablement de plus en plus besoin dans les prochaines années.

\section{Avenir du commerce du bétail.}

Le partage de l'Afrique en grandes zones climatıques très marquées et le développement rapide des grandes villes et des pofulations rurales des pays côtiers les moıns bien pourvus en bétail, ont provoqué un système orıgınal d'approvisionnement en animaux, dont l'importance économique est beaucoup plus grande qu'on ne l'imagine souvent.

Les tableaux Nas 11 ef 12 donnent les ordres de grandeur du nombre de têtes de bovins faisant l'objet de ce commerce.

\section{TABLEAU No 11}

Importance des abattages dans les grandes villes africaines ef molgaches

Untté : nombre de bovins chiffres arrondis.

\begin{tabular}{|c|c|c|c|c|}
\hline \multicolumn{3}{|c|}{ Afrique de l'Ouest } & \multicolumn{2}{|c|}{$\begin{array}{l}\text { Madogascar } \\
\text { (année 1960) }\end{array}$} \\
\hline $\begin{array}{c}\text { Centre } \\
\text { consıdéré }\end{array}$ & $\begin{array}{l}\text { Nbre de } \\
\text { bovins } \\
\text { abattus }\end{array}$ & $\begin{array}{l}\text { Année } \\
\text { de ré- } \\
\text { férence }\end{array}$ & $\begin{array}{c}\text { Centres } \\
\text { considérés }\end{array}$ & $\begin{array}{c}\text { Nbre de } \\
\text { bovins } \\
\text { abattus }\end{array}$ \\
\hline $\begin{array}{l}\text { Dakar ..... } \\
\text { Abidjan ..... } \\
\text { Bauaké ..... } \\
\text { Bamako .... } \\
\text { Ouagadougou } \\
\text { Bobodioulasso } \\
\text { Accra ...... } \\
\text { Niamey .... } \\
\text { Fort Lamy ... } \\
\text { Bangui ..... } \\
\text { Douala .... } \\
\text { Yaoundé ... }\end{array}$ & $\begin{array}{l}40.100 \\
31.500 \\
10.500 \\
35.000 \\
24.700 \\
15.000 \\
27200 \\
25.000 \\
39.700 \\
27.700 \\
13.300 \\
11.600\end{array}$ & $\begin{array}{l}1965 \\
1964 \\
1964 \\
1964 \\
1964 \\
1964 \\
1964 \\
1964 \\
1965 \\
1966 \\
1963 \\
1963\end{array}$ & $\begin{array}{l}\text { Tananarıve . } \\
\text { Tamatave ... } \\
\text { Majunga ... } \\
\text { Fuanarantsoa } \\
\text { Tuléar ..... } \\
\text { Diégosuarez } \\
\text { Antsirabé ... } \\
\text { Fort Dauphin } \\
\text { Ambositra ... } \\
\text { Morovoay... } \\
\text { Moroudava. } \\
\text { Hell Ville ... } \\
\text { Ambaloudra- } \\
\text { zaka ...... }\end{array}$ & $\begin{array}{r}35.600 \\
6300 \\
6800 \\
5.000 \\
3900 \\
4.600 \\
5.700 \\
1.200 \\
2000 \\
1.500 \\
1.000 \\
1.000 \\
\\
1.600\end{array}$ \\
\hline Total .... & 300.300 & & Total..... & 76.200 \\
\hline
\end{tabular}

On peut donc estimer que les très grands circuits portent à eux seuls, aujourd'hui, sur sept cents à huit cent mille têtes de bovins, ce qui est considérable et représente de cent d cent vingt cing mille tonnes de viandes en carcasses.

La valeur de ce cheptel chez le producteur est de l'ordre de neuf à onze mulliards de francs CFA, et il faut bien soulıgner que nous n'avons considéré icı que les échanges Inter-états. II faudrait
TABLEAU Na 12

Importance des grands courants des échanges inter-Etats en Afrique de l'Ouest

Unıtés : nombre de bovins chiffres arrondis.

\begin{tabular}{|c|c|c|c|}
\hline $\begin{array}{c}\text { Pays } \\
\text { exportateur }\end{array}$ & $\begin{array}{c}\text { Pays } \\
\text { Importateur }\end{array}$ & $\begin{array}{l}\text { Nombre } \\
\text { de têtes } \\
\text { de bovins }\end{array}$ & $\begin{array}{c}\text { Total } \\
\text { des sorties } \\
\text { des pays } \\
\text { exportateurs }\end{array}$ \\
\hline Mauritanie & $\begin{array}{l}\text { Sénégal ...... } \\
\text { Mali ....... }\end{array}$ & $\begin{array}{l}60-70.000 \\
15-20.000\end{array}$ & 75 à 90.000 \\
\hline Malı & $\begin{array}{l}\text { Côte d'Ivolre.. } \\
\text { Ghana........ } \\
\text { Frontaliers el } \\
\text { viandes forai- } \\
\text { nes ......... }\end{array}$ & $\begin{array}{r}80-90000 \\
60-70.000 \\
15-20000\end{array}$ & 155 d̀ 180.000 \\
\hline Haute Volta & $\begin{array}{l}\text { Côte d'lvoire. } \\
\text { Ghana........ } \\
\text { Frontaliers et } \\
\text { viandes foral- } \\
\text { nes ......... }\end{array}$ & $\begin{array}{r}25-30.000 \\
35-40.000 \\
5-10000\end{array}$ & 65 d̀ 80.000 \\
\hline Niger & $\begin{array}{l}\text { Nigérı ...... } \\
\text { Divers fronta- } \\
\text { liers et viandes } \\
\text { foraınes ..... }\end{array}$ & $\begin{array}{r}150-160.000 \\
20-30.000\end{array}$ & 170 à 180000 \\
\hline Tchad & $\begin{array}{l}\text { Nigéria } \ldots . . \\
\text { R. C. A. ..... } \\
\text { Cameroun .... } \\
\text { Viandes foral- } \\
\text { nes ......... }\end{array}$ & $\begin{array}{r}150-170.000 \\
50-60.000 \\
10-20000 \\
25-30.000\end{array}$ & 235 d̀ 280000 \\
\hline Total ... & & & 700 d̀ 810.000 \\
\hline
\end{tabular}

donc, pour avolr une idée de l'importance du commerce du bétail, ajouter la valeur du cheptel faisant l'objet de transactions pour les divers besoins intérieurs; nous l'évaluons très approximativement à la moitié de la précédente. Le montant annuel des achats de bétail chez les producteurs seralt donc, dans la zone considérée, de l'ordre de quatorze à dix-sept milliards de F. CFA. Sı l'on considère que, pour obtenir le coût du bétail rendu dans les centres consommateurs, on doit majorer le prix producteur de 30 p. 100 pour les marchés intérieurs et de 50 p. 100 pour les circuits longs, le montant de l'ensemble des transactions da la vente sur les marchés de bétail des villes de consommation serait de l'ordre de vingt à vingt-quatre milliards de F. CFA.

A titre d'information, on peut indiquer que la valeur finale de la viande et des abats tirés de ce bétail, après transformation et vente au détail, se trouve majorée d'environ vingt-cing pour cent. 
II nous paraît intéressant de noter qu'un commerce d'une telle ampleur, en grande majorité de type traditionnel, ait pu, sans apport financier, ni alde bancaıre extérieure, faıre face durant les années passées à la demande et à son impressionnant développement et ce, dans un milieu partıculièrement difficile et malgré les très mauvaises conditions de paiements sur les marchés de consommation.

Ces considérations économıques et la certıtude que le volume des transactions devra s'accroître régulıèrement d'au moıns quatre pour cent chaque année. soulignent l'ıntérêt du commerce, de bétal en Afrıque et les efforts qui méritent d'ètre entrepris pour que les circuits traditıonnels soient amélıorés dès aujourd'huı et que tout solt mis en œuvre pour permettre un développement harmonieux des méthodes modernes du service au fur et à mesure que l'infrastructure de base s'améliorera dans ces états.

\section{SUMMARY}

\section{Problems of livestock commercialization in Africa}

This paper deals with various aspecls of stock dealing in West Africa, where the produclion area is the Sahelian region, and the consumer centres are the countries situated along the Atlantic (from Senegal to the Central African Republic). Attention is also given to the relation between the producion areas and the large lowns in the regions of Cameroun and Madagascar

The majority of the animals in central tropical Africa are reared in the most northerly areas, i. e. those which, were it not for these pastoral activities, would be completely deserted by man.

Table 1 gives the numbers of stock for the various producing countries in proportion to the population density. The lowest bovine density is found in the counlries which have the highest slandard of IIving (Ivory Coast and Ghana). With respect to methods of commerce, the present situation 15 such that the problem of transporlation of the anımals to the consumer centres frequently takes precedence over efficient trading.

Tables 4 and 5 give an indication of the costs of commercialization by the two main methods of transportation, i. e. on foot and by lorry, neither of which however affects the price paid to the owner for the animal.

The advantage of modern transporl is that the anımal does not lose weight en route, which is very important, but does not mean that the producer is paid more or that the price of meat for the consumer is reduced. As far as the future is concerned, the development of meat production must be given absolute priority. To do this, some considerable problems will have to be solved, the most important of these being rearing technique ; an improvement in quality of the animals and in means of transport; and an improvement in busıness merhods combined with proper organization of meat marketing, accompanied by the necessary payment facilities at the mar cets of consumer centres

\section{RESUMEN}

Problemas de la comercialización en Africa

En este informe se traton varios aspectos concernientes al comercio del ganado mayor en Africa del Oeste, cuya zana de producción es la zona sahelıana y los centros consumıdores los paises a lo largo del oceano allántico (del Senegal a la República Centroafricana). Se nota tambien la relación entre las regiones pro. ductoras y ios grandes ciudades en Camerún y Madagascar. El mós importanle número de anımales en Africa central tropical se cria en las zonas más septentrionales, es decır en las regiones que el hombre desertaría totalmente sin ésta actividad pastoral. En el cuadro $n^{0} 1$, se dan los efectivos del ganado de los diferentes paises en relación con la densıdad de la población. La densıdad más 
baja de los bovinos se encuentra en los passes teniendo el nivel de vida más elevado (Costa de Marfil y Ghana).

En cuanto a las modalidades del comercio, según la situación existente, el problema de los transportes de animales hacia los centros de consumo a menudo domina la economia de los sistemas.

Los cuadros $n^{a} 4$ y 5 indican las costas de comercialización por dos medios principales de transporte : encaminamiento a pies y por camıon, entre los cuales casi ninguna diferencia existe en cuanto al precio del ganado vendido en la zona de consumo.

La ventaja del transporte moderno es que el animal no pierde peso durante el dicho, lo que es muy importante, pero eso no permita pagar mejor el productor o bajar el precio de la carne para el consumıdor. En el porvenir, se necesıta tener una priaridad absoluta la extensión de la producción de carne. Para eso, hay que resolver importantes problemas, entre los cuales los más importantes son : la técnica de ganaderia, la mejora de la calidad del ganado y de los medios de transporte, la mejora de las madalıdades comerciales combinada con una organización racional de la venta de la carne, acompañada por las facilidades necesarias de pago del ganado en los mercados de los centros de consumo. 